\title{
APPLICATION OF AN INTEGRAL FORMULA TO CR-SUBMANIFOLDS OF COMPLEX HYPERBOLIC SPACE
}

\author{
JIN SUK PAK AND HYANG SOOK KIM
}

Received 24 May 2004 and in revised form 2 March 2005

The purpose of this paper is to study $n$-dimensional compact CR-submanifolds of complex hyperbolic space $\mathrm{CH}^{(n+p) / 2}$, and especially to characterize geodesic hypersphere in $\mathrm{CH}^{(n+1) / 2}$ by an integral formula.

\section{Introduction}

Let $\bar{M}$ be a complex space form of constant holomorphic sectional curvature $c$ and let $M$ be an $n$-dimensional CR-submanifold of $(n-1)$ CR-dimension in $\bar{M}$. Then $M$ has an almost contact metric structure $(F, U, u, g)$ (see Section 2) induced from the canonical complex structure of $\bar{M}$. Hence on an $n$-dimensional CR-submanifold of $(n-1) \mathrm{CR}$ dimension, we can consider two structures, namely, almost contact structure $F$ and a submanifold structure represented by second fundamental form $A$. In this point of view, many differential geometers have classified $M$ under the conditions concerning those structures (cf. $[3,5,8,9,10,11,12,14,15,16])$. In particular, Montiel and Romero [12] have classified real hypersurfaces $M$ of complex hyperbolic space $\mathrm{CH}^{(n+1) / 2}$ which satisfy the commutativity condition

(C)

$$
F A=A F
$$

by using the $S^{1}$-fibration $\pi: H_{1}^{n+2} \rightarrow \mathrm{CH}^{(n+1) / 2}$ of the anti-de Sitter space $H_{1}^{n+2}$ over $\mathrm{CH}^{(n+1) / 2}$, and obtained Theorem 4.1 stated in Section 2. We notice that among the model spaces in Theorem 4.1, the geodesic hypersphere is only compact.

In this paper, we will investigate $n$-dimensional compact CR-submanifold of $(n-1)$ CR-dimension in complex hyperbolic space and provide a characterization of the geodesic hypersphere, which is equivalent to condition (C), by using the following integral formula established by Yano $[17,18]$ :

$\int_{M} \operatorname{div}\left\{\left(\nabla_{X} X\right)-(\operatorname{div} X) X\right\} * 1=\int_{M}\left\{\operatorname{Ric}(X, X)+\frac{1}{2}\left\|\mathscr{L}_{X} g\right\|^{2}-\|\nabla X\|^{2}-(\operatorname{div} X)^{2}\right\} * 1=0$, 
988 Application of an integral formula to CR-submanifolds

where $X$ is an arbitrary vector field tangent to $M$. Our results of the paper are complex hyperbolic versions of those in $[6,15]$.

\section{Preliminaries}

Let $M$ be an $n$-dimensional CR-submanifold of $(n-1)$ CR-dimension isometrically immersed in a complex space form $\bar{M}^{(n+p) / 2}(c)$. Denoting by $(J, \bar{g})$ the Kähler structure of $\bar{M}^{(n+p) / 2}(c)$, it follows by definition (cf. $[5,6,8,9,13,16]$ ) that the maximal $J$-invariant subspace

$$
\mathscr{D}_{x}:=T_{x} M \cap J T_{x} M
$$

of the tangent space $T_{x} M$ of $M$ at each point $x$ in $M$ has constant dimension $(n-1)$. So there exists a unit vector field $U_{1}$ tangent to $M$ such that

$$
\mathscr{D}_{x}^{\perp}=\operatorname{Span}\left\{U_{1}\right\}, \quad \forall x \in M,
$$

where $\mathscr{D}_{x}^{\perp}$ denotes the subspace of $T_{x} M$ complementary orthogonal to $\mathscr{D}_{x}$. Moreover, the vector field $\xi_{1}$ defined by

$$
\xi_{1}:=J U_{1}
$$

is normal to $M$ and satisfies

$$
J T M \subset T M \oplus \operatorname{Span}\left\{\xi_{1}\right\}
$$

Hence we have, for any tangent vector field $X$ and for a local orthonormal basis $\left\{\xi_{1}\right.$, $\left.\xi_{\alpha}\right\}_{\alpha=2, \ldots, p}$ of normal vectors to $M$, the following decomposition in tangential and normal components:

$$
\begin{gathered}
J X=F X+u^{1}(X) \xi_{1} \\
J \xi_{\alpha}=-U_{\alpha}+P \xi_{\alpha}, \quad \alpha=1, \ldots, p .
\end{gathered}
$$

Since the structure $(J, \bar{g})$ is Hermitian and $J^{2}=-I$, we can easily see from (2.5) and (2.6) that $F$ and $P$ are skew-symmetric linear endomorphisms acting on $T_{x} M$ and $T_{x} M^{\perp}$, respectively, and that

$$
\begin{gathered}
g\left(F U_{\alpha}, X\right)=-u^{1}(X) \bar{g}\left(\xi_{1}, P \xi_{\alpha}\right), \\
g\left(U_{\alpha}, U_{\beta}\right)=\delta_{\alpha \beta}-\bar{g}\left(P \xi_{\alpha}, P \xi_{\beta}\right),
\end{gathered}
$$

where $T_{x} M^{\perp}$ denotes the normal space of $M$ at $x$ and $g$ the metric on $M$ induced from $\bar{g}$. Furthermore, we also have

$$
g\left(U_{\alpha}, X\right)=u^{1}(X) \delta_{1 \alpha}
$$

and consequently,

$$
g\left(U_{1}, X\right)=u^{1}(X), \quad U_{\alpha}=0, \quad \alpha=2, \ldots, p
$$


Next, applying $J$ to (2.5) and using (2.6) and (2.10), we have

$$
F^{2} X=-X+u^{1}(X) U_{1}, \quad u^{1}(X) P \xi_{1}=-u^{1}(F X) \xi_{1},
$$

from which, taking account of the skew-symmetry of $P$ and (2.7),

$$
u^{1}(F X)=0, \quad F U_{1}=0, \quad P \xi_{1}=0 .
$$

Thus (2.6) may be written in the form

$$
J \xi_{1}=-U_{1}, \quad J \xi_{\alpha}=P \xi_{\alpha}, \quad \alpha=2, \ldots, p .
$$

These equations tell us that $\left(F, g, U_{1}, u^{1}\right)$ defines an almost contact metric structure on $M$ (cf. $[5,6,8,9,16]$ ), and consequently, $n=2 m+1$ for some integer $m$.

We denote by $\bar{\nabla}$ and $\nabla$ the Levi-Civita connection on $\bar{M}^{(n+p) / 2}(c)$ and $M$, respectively. Then the Gauss and Weingarten formulas are given by

$$
\begin{gathered}
\bar{\nabla}_{X} Y=\nabla_{X} Y+h(X, Y), \\
\bar{\nabla}_{X} \xi_{\alpha}=-A_{\alpha} X+\nabla_{X}^{\perp} \xi_{\alpha}, \quad \alpha=1, \ldots, p,
\end{gathered}
$$

for any vector fields $X, Y$ tangent to $M$. Here $\nabla^{\perp}$ denotes the normal connection induced from $\bar{\nabla}$ in the normal bundle $T M^{\perp}$ of $M$, and $h$ and $A_{\alpha}$ the second fundamental form and the shape operator corresponding to $\xi_{\alpha}$, respectively. It is clear that $h$ and $A_{\alpha}$ are related by

$$
h(X, Y)=\sum_{\alpha=1}^{p} g\left(A_{\alpha} X, Y\right) \xi_{\alpha} .
$$

We put

$$
\nabla_{X}^{\perp} \xi_{\alpha}=\sum_{\beta=1}^{p} s_{\alpha \beta}(X) \xi_{\beta} .
$$

Then $\left(s_{\alpha \beta}\right)$ is the skew-symmetric matrix of connection forms of $\nabla^{\perp}$.

Now, using (2.14), (2.15), and (2.17), and taking account of the Kähler condition $\bar{\nabla} J=$ 0 , we differentiate (2.5) and (2.6) covariantly and compare the tangential and normal parts. Then we can easily find that

$$
\begin{gathered}
\left(\nabla_{X} F\right) Y=u^{1}(Y) A_{1} X-g\left(A_{1} Y, X\right) U_{1}, \\
\left(\nabla_{X} u^{1}\right)(Y)=g\left(F A_{1} X, Y\right), \\
\nabla_{X} U_{1}=F A_{1} X, \\
g\left(A_{\alpha} U_{1}, X\right)=-\sum_{\beta=2}^{p} s_{1 \beta}(X) \bar{g}\left(P \xi_{\beta}, \xi_{\alpha}\right), \quad \alpha=2, \ldots, p,
\end{gathered}
$$

for any $X, Y$ tangent to $M$. 
In the rest of this paper, we suppose that the distinguished normal vector field $\xi_{1}$ is parallel with respect to the normal connection $\nabla^{\perp}$. Hence (2.17) gives

$$
s_{1 \alpha}=0, \quad \alpha=2, \ldots, p,
$$

which, together with (2.21), yields

$$
A_{\alpha} U_{1}=0, \quad \alpha=2, \ldots, p
$$

On the other hand, the ambient manifold $\bar{M}^{(n+p) / 2}(c)$ is of constant holomorphic sectional curvature $c$ and consequently, its Riemannian curvature tensor $\bar{R}$ satisfies

$$
\bar{R}_{\bar{X} \bar{Y}} \bar{Z}=\frac{c}{4}\{\bar{g}(\bar{Y}, \bar{Z}) \bar{X}-\bar{g}(\bar{X}, \bar{Z}) \bar{Y}+\bar{g}(J \bar{Y}, \bar{Z}) J \bar{X}-\bar{g}(J \bar{X}, \bar{Z}) J \bar{Y}-2 \bar{g}(J \bar{X}, \bar{Y}) J \bar{Z}\}
$$

for any $\bar{X}, \bar{Y}, \bar{Z}$ tangent to $\bar{M}^{(n+p) / 2}(c)($ cf. $[1,2,4,19])$. So, the equations of Gauss and Codazzi imply that

$$
\begin{aligned}
& R_{X Y} Z= \frac{c}{4}\{g(Y, Z) X-g(X, Z) Y+g(F Y, Z) F X-g(F X, Z) F Y-2 g(F X, Y) F Z\} \\
&+\sum_{\alpha}\left\{g\left(A_{\alpha} Y, Z\right) A_{\alpha} X-g\left(A_{\alpha} X, Z\right) A_{\alpha} Y\right\} \\
&\left(\nabla_{X} A_{1}\right) Y-\left(\nabla_{Y} A_{1}\right) X=\frac{c}{4}\left\{g\left(X, U_{1}\right) F Y-g\left(Y, U_{1}\right) F X-2 g(F X, Y) U_{1}\right\},
\end{aligned}
$$

for any $X, Y, Z$ tangent to $M$ with the aid of (2.22), where $R$ denotes the Riemannian curvature tensor of $M$. Moreover, (2.11) and (2.25) yield

$$
\begin{gathered}
\operatorname{Ric}(X, Y)=\frac{c}{4}\left\{(n+2) g(X, Y)-3 u^{1}(X) u^{1}(Y)\right\}+\sum_{\alpha}\left\{\left(\operatorname{tr} A_{\alpha}\right) g\left(A_{\alpha} X, Y\right)-g\left(A_{\alpha}^{2} X, Y\right)\right\}, \\
\rho=\frac{c}{4}(n+3)(n-1)+n^{2}\|\mu\|^{2}-\sum_{\alpha} \operatorname{tr} A_{\alpha}{ }^{2}
\end{gathered}
$$

where Ric and $\rho$ denote the Ricci tensor and the scalar curvature, respectively, and

$$
\mu=\frac{1}{n} \sum_{\alpha}\left(\operatorname{tr} A_{\alpha}\right) \xi_{\alpha}
$$

is the mean curvature vector (cf. $[1,2,4,19])$.

\section{Codimension reduction of CR-submanifolds of $\mathrm{CH}^{(n+p) / 2}$}

Let $M$ be an $n$-dimensional CR-submanifold of $(n-1)$ CR-dimension in a complex hyperbolic space $\mathrm{CH}^{(n+p) / 2}$ with constant holomorphic sectional curvature $c=-4$.

Applying the integral formula (1.2) to the vector field $U_{1}$, we have

$$
\int_{M}\left\{\operatorname{Ric}\left(U_{1}, U_{1}\right)+\frac{1}{2}\left\|\mathscr{L}_{U_{1}} g\right\|^{2}-\left\|\nabla U_{1}\right\|^{2}-\left(\operatorname{div} U_{1}\right)^{2}\right\} * 1=0 .
$$


Now we take an orthonormal basis $\left\{U_{1}, e_{a}, e_{a^{*}}\right\}_{a=1, \ldots,(n-1) / 2}$ of tangent vectors to $M$ such that

$$
e_{a^{*}}:=F e_{a}, \quad a=1, \ldots, \frac{n-1}{2} .
$$

Then it follows from (2.11) and (2.20) that

$$
\operatorname{div} U_{1}=\operatorname{tr}\left(F A_{1}\right)=\sum_{a=1}^{(n-1) / 2}\left\{g\left(F A_{1} e_{a}, e_{a}\right)+g\left(F A_{1} e_{a^{*}}, e_{a^{*}}\right)\right\}=0 .
$$

Also, using (2.20), we have

$$
\left\|\nabla U_{1}\right\|^{2}=g\left(F A_{1} U_{1}, F A_{1} U_{1}\right)+\sum_{a=1}^{(n-1) / 2}\left\{g\left(F A_{1} e_{a}, F A_{1} e_{a}\right)+g\left(F A_{1} e_{a^{*}}, F A_{1} e_{a^{*}}\right)\right\},
$$

from which, together with (2.11) and (2.12), we can easily obtain

$$
\left\|\nabla U_{1}\right\|^{2}=\operatorname{tr} A_{1}^{2}-\left\|A_{1} U_{1}\right\|^{2}
$$

Furthermore, (2.20) yields

$$
\left(\mathscr{L}_{U_{1}} g\right)(X, Y)=g\left(\nabla_{X} U_{1}, Y\right)+g\left(\nabla_{Y} U_{1}, X\right)=g\left(\left(F A_{1}-A_{1} F\right) X, Y\right),
$$

and consequently,

$$
\left\|\mathscr{L}_{U_{1}} g\right\|^{2}=\left\|F A_{1}-A_{1} F\right\|^{2}
$$

On the other hand, (2.27) and (2.28) with $c=-4$ yield

$$
\begin{gathered}
\operatorname{Ric}\left(U_{1}, U_{1}\right)=-(n-1)+u^{1}\left(A_{1} U_{1}\right)\left(\operatorname{tr} A_{1}\right)-\left\|A_{1} U_{1}\right\|^{2}, \\
\operatorname{tr}\left(A_{1}^{2}\right)=-\rho-(n+3)(n-1)+n^{2}\|\mu\|^{2}-\sum_{\alpha=2}^{p} \operatorname{tr} A_{\alpha}{ }^{2} .
\end{gathered}
$$

Substituting (3.3), (3.5), (3.7), (3.8), and (3.9) into (3.1), we have

$$
\begin{aligned}
\int_{M}\left\{\frac{1}{2}\left\|F A_{1}-A_{1} F\right\|^{2}+\operatorname{Ric}\left(U_{1}, U_{1}\right)+\rho-n^{2}\|\mu\|^{2}\right. \\
\left.+\left\|A_{1} U_{1}\right\|^{2}+(n+3)(n-1)+\sum_{\alpha=2}^{p} \operatorname{tr} A_{\alpha}^{2}\right\} * 1=0,
\end{aligned}
$$

or equivalently,

$$
\int_{M}\left\{\frac{1}{2}\left\|F A_{1}-A_{1} F\right\|^{2}+u^{1}\left(A_{1} U_{1}\right)\left(\operatorname{tr} A_{1}\right)-\operatorname{tr} A_{1}^{2}-(n-1)\right\} * 1=0 .
$$

Thus we have the following lemma. 
992 Application of an integral formula to CR-submanifolds

Lemma 3.1. Let $M$ be an n-dimensional compact orientable CR-submanifold of ( $n-1) \mathrm{CR}$ dimension in a complex hyperbolic space $\mathrm{CH}^{(n+p) / 2}$. If the distinguished normal vector field $\xi_{1}$ is parallel with respect to the normal connection and if the inequality

$$
\operatorname{Ric}\left(U_{1}, U_{1}\right)+\rho-n^{2}\|\mu\|^{2}+\left\|A_{1} U_{1}\right\|^{2}+(n+3)(n-1) \geq 0
$$

holds on $M$, then

$$
A_{1} F=F A_{1}
$$

and $A_{\alpha}=0$ for $\alpha=2, \ldots, p$.

COROLlary 3.2. Let $M$ be a compact orientable real hypersurface of $\mathrm{CH}^{(n+1) / 2}$ over which the inequality

$$
\operatorname{Ric}\left(U_{1}, U_{1}\right)+\rho-n^{2}\|\mu\|^{2}+\left\|A_{1} U_{1}\right\|^{2}+(n+3)(n-1) \geq 0
$$

holds. Then $M$ satisfies the commutativity condition (C).

Combining Lemma 3.1 and the codimension reduction theorem proved in [7, Theorem 3.2, page 126], we have the following theorem.

Theorem 3.3. Let $M$ be an $n$-dimensional compact orientable CR-submanifold of $(n-1)$ $\mathrm{CR}$-dimension in a complex hyperbolic space $\mathrm{CH}^{(n+p) / 2}$. If the distinguished normal vector field $\xi_{1}$ is parallel with respect to the normal connection and if the inequality

$$
\operatorname{Ric}\left(U_{1}, U_{1}\right)+\rho-n^{2}\|\mu\|^{2}+\left\|A_{1} U_{1}\right\|^{2}+(n+3)(n-1) \geq 0
$$

holds on $M$, then there exists a totally geodesic complex hyperbolic space $\mathrm{CH}^{(n+1) / 2}$ immersed in $\mathrm{CH}^{(n+p) / 2}$ such that $M \subset \mathrm{CH}^{(n+1) / 2}$. Moreover $M$ satisfies the commutativity condition (C) as a real hypersurface of $\mathrm{CH}^{(n+1) / 2}$.

Proof. Let

$$
N_{0}(x):=\left\{\eta \in T_{x} M^{\perp} \mid A_{\eta}=0\right\}
$$

and let $H_{0}(x)$ be the maximal holomorphic subspace of $N_{0}(x)$, that is,

$$
H_{0}(x)=N_{0}(x) \cap J N_{0}(x)
$$

Then, by means of Lemma 3.1,

$$
H_{0}(x)=N_{0}(x)=\operatorname{Span}\left\{\xi_{2}, \ldots, \xi_{p}\right\}
$$

Hence, the orthogonal complement $H_{1}(x)$ of $H_{0}(x)$ in $T M^{\perp}$ is $\operatorname{Span}\left\{\xi_{1}\right\}$ and so, $H_{1}(x)$ is invariant under the parallel translation with respect to the normal connection and $\operatorname{dim} H_{1}(x)=1$ at any point $x \in M$. Thus, applying the codimension reduction theorem in [4] proved by Kawamoto, we verify that there exists a totally geodesic complex hyperbolic space $\mathrm{CH}^{(n+1) / 2}$ immersed in $\mathrm{CH}^{(n+p) / 2}$ such that $M \subset \mathrm{CH}^{(n+1) / 2}$. Therefore, $M$ can 
be regarded as a real hypersurface of $\mathrm{CH}^{(n+1) / 2}$ which is totally geodesic in $\mathrm{CH}^{(n+p) / 2}$. Tentatively, we denote $\mathrm{CH}^{(n+1) / 2}$ by $M^{\prime}$, and by $i_{1}$ we denote the immersion of $M$ into $M^{\prime}$, and by $i_{2}$ the totally geodesic immersion of $M^{\prime}$ into $\mathrm{CH}^{(n+p) / 2}$. Then it is clear from $(2.14)$ that

$$
\nabla_{i_{1} X}^{\prime} i_{1} Y=i_{1} \nabla_{X} Y+h^{\prime}(X, Y)=i_{1} \nabla_{X} Y+g\left(A^{\prime} X, Y\right) \xi^{\prime}
$$

where $\nabla^{\prime}$ is the induced connection on $M^{\prime}$ from that of $\mathrm{CH}^{(n+p) / 2}, h^{\prime}$ the second fundamental form of $M$ in $M^{\prime}$, and $A^{\prime}$ the corresponding shape operator to a unit normal vector field $\xi^{\prime}$ to $M$ in $M^{\prime}$. Since $i=i_{2} \circ i_{1}$ and $M^{\prime}$ is totally geodesic in $\mathrm{CH}^{(n+p) / 2}$, we can easily see that (2.15) and (3.19) imply that

$$
\xi_{1}=i_{2} \xi^{\prime}, \quad A_{1}=A^{\prime} .
$$

Since $M^{\prime}$ is a holomorphic submanifold of $\mathrm{CH}^{(n+p) / 2}$, for any $X$ in $T M$,

$$
J i_{2} X=i_{2} J^{\prime} X
$$

is valid, where $J^{\prime}$ is the induced Kähler structure on $M^{\prime}$. Thus it follows from (2.5) that

$$
\begin{aligned}
J i X & =J i_{2} \circ i_{1} X=i_{2} J^{\prime} i_{1} X=i_{2}\left(i_{1} F^{\prime} X+u^{\prime}(X) \xi^{\prime}\right) \\
& =i F^{\prime} X+u^{\prime}(X) i_{2} \xi^{\prime}=i F^{\prime} X+u^{\prime}(X) \xi_{1}
\end{aligned}
$$

for any vector field $X$ tangent to $M$. Comparing this equation with (2.5), we have $F=F^{\prime}$ and $u^{1}=u^{\prime}$, which, together with Lemma 3.1, implies that

$$
A^{\prime} F^{\prime}=F^{\prime} A^{\prime}
$$

\section{An integral formula on the model space $M_{2 p+1,2 q+1}^{h}(r)$}

We first explain the model hypersurfaces of complex hyperbolic space due to Montiel and Romero for later use (for the details, see [12]).

Consider the complex $(n+3) / 2$-space $C_{1}^{(n+3) / 2}$ endowed with the pseudo-Euclidean metric $g_{0}$ given by

$$
g_{0}=-d z_{0} d \bar{z}_{0}+\sum_{j=1}^{m} d z_{j} d \bar{z}_{j}, \quad\left(m+1:=\frac{n+3}{2}\right),
$$

where $\bar{z}_{k}$ denotes the complex conjugate of $z_{k}$.

On $C_{1}^{(n+3) / 2}$, we define

$$
F(z, w)=-z_{0} \bar{w}_{0}+\sum_{k=1}^{m} z_{k} \bar{w}_{k}
$$

Put

$$
H_{1}^{n+2}=\left\{z=\left(z_{0}, z_{1}, \ldots, z_{m}\right) \in C_{1}^{(n+3) / 2}:\langle z, z\rangle=-1\right\},
$$


where $\langle\cdot, \cdot\rangle$ denotes the inner product on $C_{1}^{(n+3) / 2}$ induced from $g_{0}$. Then it is known that $H_{1}^{n+2}$, together with the induced metric, is a pseudo-Riemannian manifold of constant sectional curvature -1 , which is known as an anti-de Sitter space. Moreover, $H_{1}^{n+2}$ is a principal $S^{1}$-bundle over $\mathrm{CH}^{(n+1) / 2}$ with projection $\pi: H_{1}^{n+2} \rightarrow \mathrm{CH}^{(n+1) / 2}$ which is a Riemannian submersion with fundamental tensor $J$ and time-like totally geodesic fibers.

Given $p, q$ integers with $2 p+2 q=n-1$ and $r \in R$ with $0<r<1$, we denote by $M_{2 p+1,2 q+1}(r)$ the Lorentz hypersurface of $H_{1}^{n+2}$ defined by the equations

$$
-\left|z_{0}\right|^{2}+\sum_{k=1}^{m}\left|z_{k}\right|^{2}=-1, \quad r\left(-\left|z_{0}\right|^{2}+\sum_{k=1}^{p}\left|z_{k}\right|^{2}\right)=-\sum_{k=p+1}^{m}\left|z_{k}\right|^{2}
$$

where $z=\left(z_{0}, z_{1}, \ldots, z_{m}\right) \in C_{1}^{(n+3) / 2}$. In fact, $M_{2 p+1,2 q+1}(r)$ is isometric to the product

$$
H_{1}^{2 p+1}\left(\frac{1}{r-1}\right) \times S^{2 q+1}\left(\frac{r}{1-r}\right)
$$

where $1 /(r-1)$ and $r /(1-r)$ denote the squares of the radii and each factor is embedded in $H_{1}^{n+2}$ in a totally umbilical way. Since $M_{2 p+1,2 q+1}(r)$ is $S^{1}$-invariant, $M_{2 p+1,2 q+1}^{h}(r):=$ $\pi\left(M_{2 p+1,2 q+1}(r)\right)$ is a real hypersurface of $\mathrm{CH}^{(n+1) / 2}$ which is complete and satisfies the condition (C).

As already mentioned in Section 1, Montiel and Romero [12] have classified real hypersurfaces $M$ of $\mathrm{CH}^{(n+1) / 2}$ which satisfy the condition (C) and obtained the following classification theorem.

THEOREM 4.1. Let $M$ be a complete real hypersurface of $\mathrm{CH}^{(n+1) / 2}$ which satisfies the condition $(C)$. Then there exist the following possibilities.

(1) $M$ has three constant principal curvatures $\tanh \theta, \operatorname{coth} \theta, 2 \operatorname{coth} 2 \theta$ with multiplicities $2 p, 2 q, 1$, respectively, $2 p+2 q=n-1$. Moreover, $M$ is congruent to $M_{2 p+1,2 q+1}^{h}$ $\left(\tanh ^{2} \theta\right)$.

(2) $M$ has two constant principal curvatures $\lambda_{1}, \lambda_{2}$ with multiplicities $n-1$ and 1 , respectively. (i) If $\lambda_{1}>1$, then $\lambda_{1}=\operatorname{coth} \theta, \lambda_{2}=2 \operatorname{coth} 2 \theta$ with $\theta>0$, and $M$ is congruent to a geodesic hypersphere $M_{1, n}^{h}\left(\tanh ^{2} \theta\right)$. (ii) If $\lambda_{1}<1$, then $\lambda_{1}=\tanh \theta, \lambda_{2}=2 \operatorname{coth} 2 \theta$ with $\theta>0$, and $M$ is congruent to $M_{n, 1}^{h}\left(\tanh ^{2} \theta\right)$. (iii) If $\lambda_{1}=1$, then $\lambda_{2}=2$ and $M$ is congruent to a horosphere.

Combining Corollary 3.2 and Theorem 4.1, we have the following theorem.

THEOREM 4.2. Let $M$ be a compact orientable real hypersurface of $\mathrm{CH}^{(n+1) / 2}$ over which the inequality

$$
\operatorname{Ric}\left(U_{1}, U_{1}\right)+\rho-n^{2}\|\mu\|^{2}+\left\|A_{1} U_{1}\right\|^{2}+(n+3)(n-1) \geq 0
$$

holds. Then $M$ is congruent to a geodesic hypersphere $M_{1, n}^{h}(r)$ in $\mathrm{CH}^{(n+1) / 2}$.

Combining Theorems 3.3 and 4.2, we have the following theorem. 
Theorem 4.3. Let $M$ be an n-dimensional compact orientable CR-submanifold of $(n-1)$ $\mathrm{CR}$-dimension in a complex hyperbolic space $\mathrm{CH}^{(n+p) / 2}$. If the distinguished normal vector field $\xi_{1}$ is parallel with respect to the normal connection and if the inequality

$$
\operatorname{Ric}\left(U_{1}, U_{1}\right)+\rho-n^{2}\|\mu\|^{2}+\left\|A_{1} U_{1}\right\|^{2}+(n+3)(n-1) \geq 0
$$

holds on $M$, then $M$ is congruent to a geodesic hypersphere $M_{1, n}^{h}\left(\tanh ^{2} \theta\right)$ in $\mathrm{CH}^{(n+1) / 2}$.

Remark 4.4. As already shown in (3.10) and (3.11), the equality

$$
\begin{gathered}
\operatorname{Ric}\left(U_{1}, U_{1}\right)+\rho-n^{2}\|\mu\|^{2}+\left\|A_{1} U_{1}\right\|^{2}+(n+3)(n-1) \\
=u^{1}\left(A_{1} U_{1}\right)\left(\operatorname{tr} A_{1}\right)-\operatorname{tr} A_{1}^{2}-(n-1)
\end{gathered}
$$

holds on $M$. On the other hand, the geodesic hypersphere $M_{1, n}^{h}\left(\tanh ^{2} \theta\right)$ in Theorem 4.1 has constant principal curvatures $\operatorname{coth} \theta$ and $2 \operatorname{coth} 2 \theta$ with multiplicities $n-1$ and 1 , respectively. Hence we can easily verify the equality

$$
u^{1}\left(A_{1} U_{1}\right)\left(\operatorname{tr} A_{1}\right)-\operatorname{tr} A_{1}^{2}-(n-1)=0
$$

and consequently,

$$
\operatorname{Ric}\left(U_{1}, U_{1}\right)+\rho-n^{2}\|\mu\|^{2}+\left\|A_{1} U_{1}\right\|^{2}+(n+3)(n-1)=0
$$

on $M_{1, n}^{h}\left(\tanh ^{2} \theta\right)$.

Remark 4.5. If we put $V:=\nabla_{U_{1}} U_{1}-\left(\operatorname{div} U_{1}\right) U_{1}$, then it easily follows from (2.11) that $V=F A_{1} U_{1}$. Taking account of (3.3), (3.5), (3.7), and (3.8), we obtain

$$
\operatorname{div} V=\frac{1}{2}\left\|F A_{1}-A_{1} F\right\|^{2}+u^{1}\left(A_{1} U_{1}\right)\left(\operatorname{tr} A_{1}\right)-\operatorname{tr} A_{1}^{2}-(n-1) .
$$

Hence if the commutativity condition (C) holds on $M$, then the vector field $V$ is zero since $U_{1}$ is a principal vector of $A_{1}$, and consequently,

$$
u^{1}\left(A_{1} U_{1}\right)\left(\operatorname{tr} A_{1}\right)-\operatorname{tr} A_{1}^{2}-(n-1)=0 .
$$

Thus, on $n$-dimensional CR-submanifold $M$ of $(n-1)$ CR-dimension in a complex hyperbolic space $\mathrm{CH}^{(n+p) / 2}$ over which the commutativity condition $C$ holds, the function $u^{1}\left(A_{1} U_{1}\right)$ cannot be zero at any point of $M$. A real hypersurface of a complex hyperbolic space $\mathrm{CH}^{(n+p) / 2}$ satisfying the commutativity condition (C) cannot be minimal.

\section{Acknowledgment}

This work was supported by ABRL Grant Project no. R14-2002-003-01000-0 from Korea Science and Engineering Foundation (KOSEF). 


\section{References}

[1] A. Bejancu, CR submanifolds of a Kaehler manifold. I, Proc. Amer. Math. Soc. 69 (1978), no. 1, $135-142$.

[2] Geometry of CR-Submanifolds, Mathematics and its Applications (East European Series), vol. 23, D. Reidel Publishing, Dordrecht, 1986.

[3] A. A. Borisenko, On the global structure of Hopf hypersurfaces in a complex space form, Illinois J. Math. 45 (2001), no. 1, 265-277.

[4] B. Y. Chen, Geometry of Submanifolds, Marcel Dekker, New York, 1973.

[5] Y.-W. Choe and M. Okumura, Scalar curvature of a certain CR-submanifold of complex projective space, Arch. Math. (Basel) 68 (1997), no. 4, 340-346.

[6] M. Djorić and M. Okumura, Certain application of an integral formula to CR-submanifold of complex projective space, Publ. Math. Debrecen 62 (2003), no. 1-2, 213-225.

[7] S. Kawamoto, Codimension reduction for real submanifolds of a complex hyperbolic space, Rev. Mat. Univ. Complut. Madrid 7 (1994), no. 1, 119-128.

[8] J.-H. Kwon and J. S. Pak, CR-submanifolds of $(n-1)$ CR-dimension in a complex projective space, Saitama Math. J. 15 (1997), 55-65.

[9] , n-dimensional CR-submanifolds of $(n-1)$ CR-dimension immersed in a complex space form, Far East J. Math. Sci., Special volume (1999), Part III, 347-360.

[10] H. B. Lawson Jr., Rigidity theorems in rank-1 symmetric spaces, J. Differential Geom. 4 (1970), 349-357.

[11] S. Montiel, Real hypersurfaces of a complex hyperbolic space, J. Math. Soc. Japan 37 (1985), no. 3, 515-535.

[12] S. Montiel and A. Romero, On some real hypersurfaces of a complex hyperbolic space, Geom. Dedicata 20 (1986), no. 2, 245-261.

[13] R. Nirenberg and R. O. Wells Jr., Approximation theorems on differentiable submanifolds of a complex manifold, Trans. Amer. Math. Soc. 142 (1969), 15-35.

[14] M. Okumura, On some real hypersurfaces of a complex projective space, Trans. Amer. Math. Soc. 212 (1975), 355-364.

[15] _ Compact real hypersurfaces of a complex projective space, J. Differential Geom. 12 (1977), no. 4, 595-598 (1978).

[16] M. Okumura and L. Vanhecke, $n$-dimensional real submanifolds with ( $n-1)$-dimensional maximal holomorphic tangent subspace in complex projective spaces, Rend. Circ. Mat. Palermo (2) 43 (1994), no. 2, 233-249.

[17] K. Yano, On harmonic and Killing vector fields, Ann. of Math. (2) 55 (1952), 38-45.

[18] , Integral Formulas in Riemannian Geometry, Pure and Applied Mathematics, vol. 1, Marcel Dekker, New York, 1970.

[19] K. Yano and M. Kon, CR Submanifolds of Kaehlerian and Sasakian Manifolds, Progress in Mathematics, vol. 30, Birkhäuser Boston, Massachusetts, 1983.

Jin Suk Pak: Department of Mathematics, College of Natural Science, Kyungpook National University, Daegu 702-701, Korea

E-mail address: jspak@knu.ac.kr

Hyang Sook Kim: Department of Computational Mathematics, School of Computer Aided Science, College of Natural Science, Inje University, Kimhae, Kyungnan 621-749, Korea

E-mail address: mathkim@inje.ac.kr 


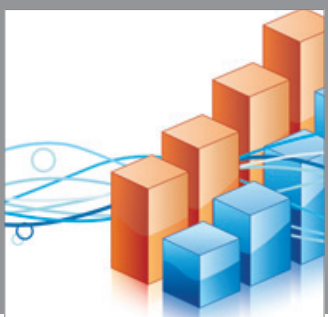

Advances in

Operations Research

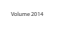

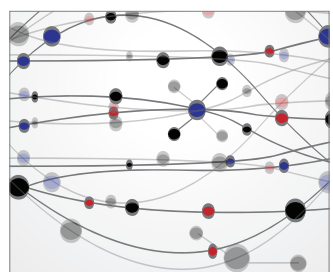

\section{The Scientific} World Journal
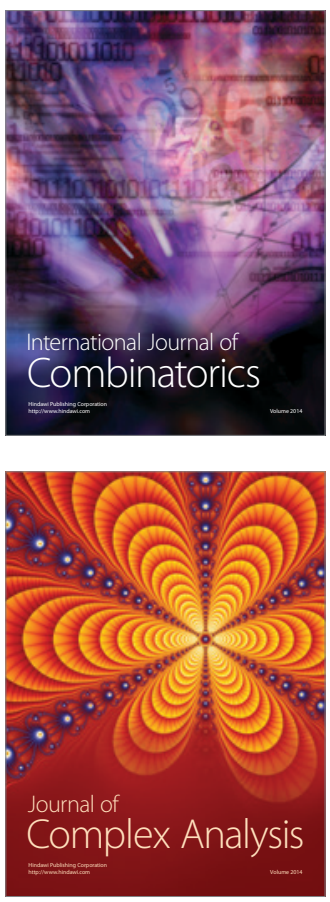

International Journal of

Mathematics and

Mathematical

Sciences
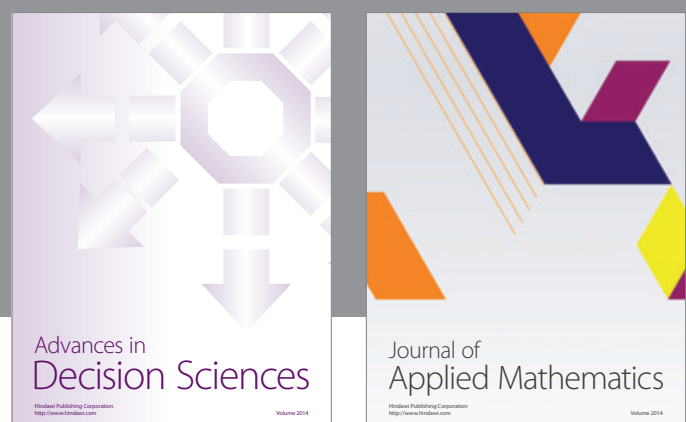

Journal of

Applied Mathematics
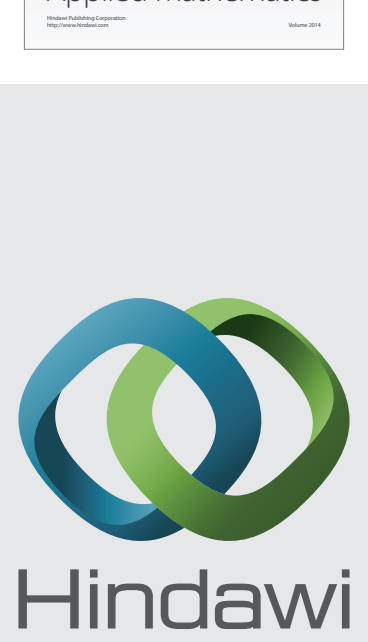

Submit your manuscripts at http://www.hindawi.com
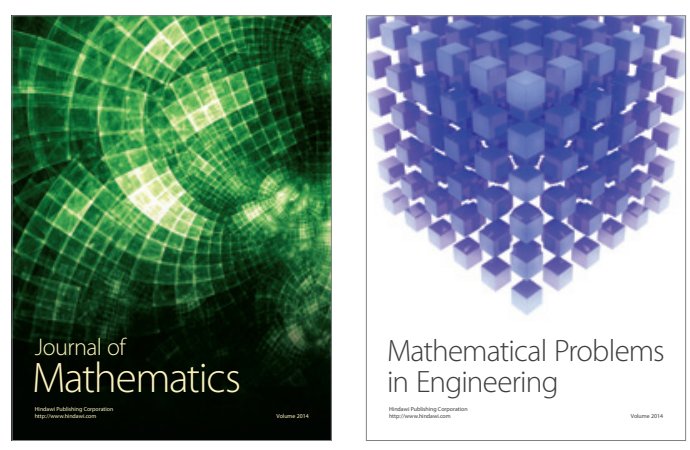

Mathematical Problems in Engineering
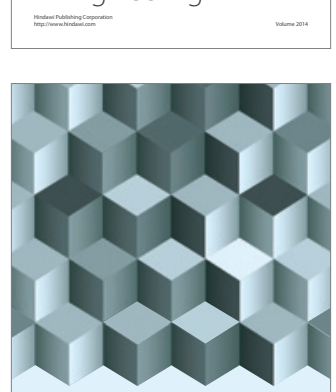

Journal of

Function Spaces
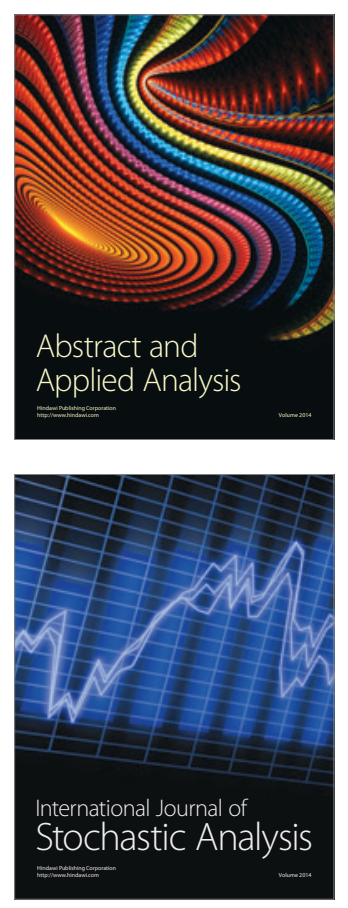

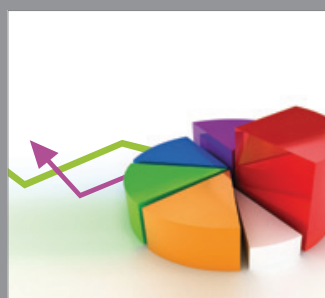

ournal of

Probability and Statistics

Promensencen
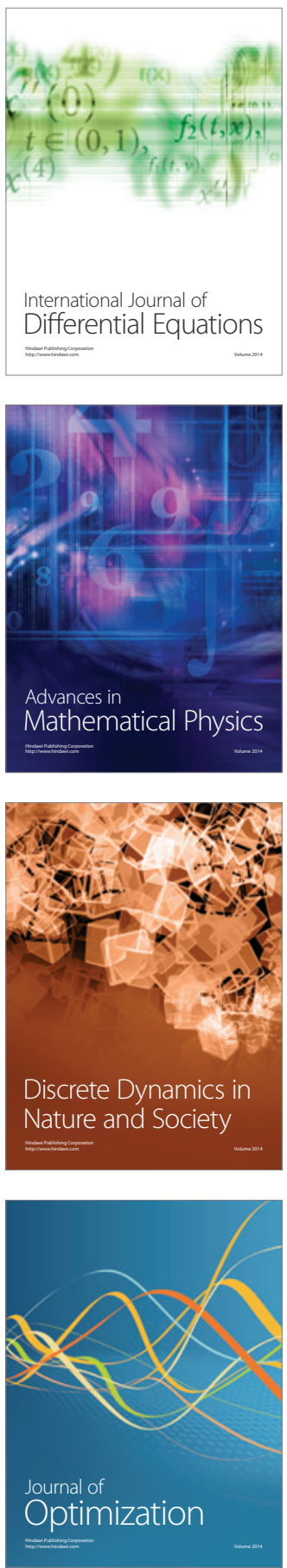